\title{
The complementary benefit of anterior segment optical coherence tomography in penetrating keratoplasty
}

This article was published in the following Dove Press journal:

Clinical Ophthalmology

22 July 2013

Number of times this article has been viewed

\section{Nursal Melda Yenerel' \\ Raciha Beril Kucumen ${ }^{2}$ \\ Ebru Gorgun ${ }^{3}$}

'Haydarpasa Numune Research and Training Hospital, Istanbul, Turkey; ${ }^{2}$ Yeditepe University Medical Faculty, Department of Ophthalmology, Istanbul, Turkey; ${ }^{3}$ Dunya Goz Eye Hospital, Istanbul, Turkey
Correspondence: Nursal Melda Yenerel Haydarpasa Numune Research and Training Hospital, Tıbbiye Caddesi, No 40 Uskudar, Istanbul, Turkey

$\mathrm{Tel}+905323258650$

Fax +90 2I2 2II 2500

Email meldayen@hotmail.com
Purpose: To evaluate the utility of anterior segment optical coherence tomography (AS-OCT) assessment in the pre- and postsurgical management of full-thickness corneal grafts.

Methods: Seventy eyes of 58 patients who had penetrating keratoplasty were included in the study. High resolution AS-OCT scans of the cornea and the anterior segment were performed before and after transplantation in 17 eyes with the Visante ${ }^{\mathrm{TM}}$ optical coherence tomography. Fifty-three eyes were examined in the late postoperative period.

Results: The mean follow-up was 4.3 years (range, 1-15 years). Six different graft-host junctions were identified. Three patients with graft rejection, four patients with anterior synechia, and three patients with corneal ulcer were evaluated. Preoperative evaluation of eyes with semiopaque and totally opaque corneas revealed important information about anterior segment structures additional to slit-lamp findings.

Conclusion: AS-OCT is a useful complementary tool for the evaluation of penetrating corneal transplantation surgery and in the management of its postoperative complications.

Keywords: anterior segment optical coherence tomography, imaging techniques, penetrating keratoplasty

\section{Introduction}

Penetrating keratoplasty (PKP) is one of the most successful tissue transplants compared with transplantation of other tissues. Advances in microsurgical techniques have allowed the indications for keratoplasty to expand considerably. ${ }^{1,2}$ Although recently developed anterior and posterior lamellar procedures such as deep anterior lamellar keratoplasty, deep lamellar endothelial keratoplasty, and Descemet's stripping automated endothelial keratoplasty are evolving and gaining popularity, PKP still remains the primary surgical approach for extremely pathologic and deformed corneas in many corneal diseases.

The success of PKP depends mostly on graft quality, operative equipment, and surgical experience, but careful postoperative surveillance is also critical for the prevention of graft failure. For this purpose, imaging of the anterior segment may play an important role in recognizing changes that may be otherwise unnoticeable during routine ophthalmological examination, and it may also give additional information to slit-lamp findings.

Anterior segment optical coherence tomography (AS-OCT) is an imaging technique that allows cross-sectional, clear visualization of the anterior segment, enabling qualitative and quantitative analysis. AS-OCT is a patient- and user-friendly noncontact procedure, which is useful in identifying anterior segment pathologies. 
This assessment can be performed preoperatively in addition to being performed in the early and late postoperative period to monitor the eye for prompt diagnosis of complications, and therefore can be very useful to the anterior segment surgeon. ${ }^{3,4}$ Recent studies investigating the utility of AS-OCT in glaucoma, after phacoemulsification, lamellar corneal surgery, during femtosecond assisted laser in situ keratomileusis, and in diverse ocular pathologies indicate that this imaging technique provides valuable information for ophthalmologists..$^{5-16}$

Our aim in this study was to demonstrate the practical use of high-resolution AS-OCT for evaluating the cornea after PKP. We report our findings in a series of patients with keratoconus, bullous keratopathy, corneal dystrophies, and corneal scars due to different etiologies. Complicated cases were closely monitored by AS-OCT during the treatment course. Routine PKP cases without complications were also examined by AS-OCT for better evaluation of the graft-host relationship in the postoperative period.

\section{Methods}

For all the study procedures, the tenets of the Declaration of Helsinki were followed; patients were informed about their inclusion in the study and gave their consent.

This study consisted of 70 eyes of 58 patients ( 16 females, 42 males) with a mean age of $42.7 \pm 17.6$ years (range, $16-88$ years). In 17 patients who were operated on in the past 3 years, both preoperative and postoperative examinations were performed; whereas in 41 patients who were in their late postoperative period (4-15 years), only the postoperative examinations could be carried out since this new technology was not available at the time of the operation. The leading indications for PKP, in decreasing order were, keratoconus (36 eyes), pseudophakic bullous keratopathy (PBK, 13 eyes), corneal dystrophy (seven eyes), trauma (five eyes), graft rejection (three eyes), sclerocornea (two eyes), pellucid marginal degeneration (one eye), herpetic keratitis (one eye), toxic anterior segment syndrome (one eye), and iatrogenic keratectasia (one eye). The mean follow-up period was 4.3 years (range, $1-15$ years).

\section{Surgical technique}

All patients were operated on by a single experienced surgeon (RBK) and were under general or local anesthesia. The optical axis was marked and the cornea was trephined using a disposable vacuum trephine system (Barron trephine system, Katena Products Inc, Denville, NJ, USA). The excision of the host cornea was completed using beveled corneal scissors. The decision for the diameter of trephination was based on the vertical corneal diameter (VCD); a $7.5 \mathrm{~mm}$ recipient trephine for $\mathrm{VCD}<10.5 \mathrm{~mm}$ and an $8.0 \mathrm{~mm}$ trephine for $\mathrm{VCD} \geq 10.5 \mathrm{~mm}$ were used. In keratoconus, a $0.25 \mathrm{~mm}$ oversized graft was preferred whereas a $0.50 \mathrm{~mm}$ oversized donor button was prepared for other pathologies. Combined surgery with anterior segment reconstruction, cataract extraction, anterior vitrectomy with intraocular lens (IOL) exchange and/or aphakia correction with transscleral fixated IOLs were performed in three eyes. The donor cornea was prepared from the endothelial side with the Barron donor punch and then placed in the recipient bed. Four cardinal interrupted sutures of 10/0 nylon were tied 90 degrees apart. In highly vascularized corneas, 16 interrupted sutures were preferred (14 eyes). In the rest of the eyes, a single running suture was placed and interrupted sutures were added where necessary. After suture tightening, control of astigmatism, and suture adjustment, the operation was completed.

\section{Anterior segment OCT analysis}

Following ophthalmological examination, eyes were evaluated using AS-OCT (Visante ${ }^{\mathrm{TM}}$ OCT; Carl Zeiss Meditec, AG, Jena, Germany). The Visante ${ }^{\mathrm{TM}}$ OCT is a high-resolution tomographic device designed for anterior segment imaging and measurement. The instrument uses the principle of low-coherence interferometry utilizing a $1310 \mathrm{~nm}$ superluminescent light-emitting diode as the light source. Its scan rate reaches up to $2048 \mathrm{~A}$-scans per second, and its axial and transverse image resolutions are up to $18 \mu \mathrm{m}$ and $60 \mu \mathrm{m}$ (Visante $^{\mathrm{TM}}$ OCT User Manual, 2006). It acquires multiple A-scans and aligns them to construct two-dimensional images similar to an ultrasound B-scan. During examination, four equally spaced radial scans through the corneal vertex were carried out for each eye with fixation monitored throughout. The reflection from the anterior vertex of the cornea saturates the system, and this was visualized as a central, vertical, highly reflective line-vertical flare. However, this line could not always be visualized in highly pathologic corneas. The Visante ${ }^{\mathrm{TM}}$ OCT has three main imaging modalities: anterior segment, high resolution cornea, and pachymetry. The anterior segment and high resolution cornea images can be obtained as single scans in the desired axis and as four synchronized scans $45^{\circ}$ apart (Visante OCT Software version 1.2.0.1).

To reduce variability, a single OCT-experienced ophthalmologist (RBK) performed all anterior segment and high-resolution corneal scans. For the purpose of this study, 
the recorded images were evaluated by two of the authors (RBK and NMY).

\section{Results}

\section{Preoperative evaluation}

From the 17 eyes of 17 patients who were examined preoperatively, six patients had keratoconus, five patients had PBK, two patients had a traumatic scar, two patients had corneal ulcer, one patient had herpetic scar, and one patient had graft failure as preoperative diagnosis. The slit-lamp examination was satisfactory in the preoperative evaluation of the anterior segment in the keratoconus patients and in the eye with herpetic scar. The pachymetry map of AS-OCT allowed us to detect the localization of the thinnest corneal area (Figure 1). In semiopaque corneas with PBK, structures of the anterior segment could be assessed, although not clearly, by slit-lamp examination. In these cases, AS-OCT imaging confirmed our assessment. On the other hand, in totally opaque corneas (six eyes) where only the outer corneal surface was visible by the slit-lamp, AS-OCT was supportive in evaluating the posterior surface of the cornea, iridocorneal angle, iris, pupil, and lens. The presence of the retrocorneal membrane, synechia, iris, and pupil abnormalities, as well as the existence of an IOL and its position could be evaluated in the anterior segment quad images (Figures 2 and 3). A high-resolution modality was utilized for the assessment of finer details.

\section{Postoperative evaluation}

In high-resolution AS-OCT images, almost all of the eyes showed a uniform anterior corneal surface indicating good congruity of the donor and host corneas (68 eyes; 97.2\%). However, the posterior corneal surface showed incongruities at the wound site in some scans, hence the internal graft-host junctions were variable. Eight graft-host junctions of 4-Quad high resolution AS-OCT images were evaluated for each eye. We have seen that a minimum of two or more variations of the internal graft-host junction could be identified per eye. The following variations were observed:

1. The thickness of the graft was equal to the thickness of the host cornea at the graft-host junction; the posterior surface of the donor button was continuous and well apposed enough with the posterior surface of the host cornea. This donor-graft relation was seen in 39 (55.7\%) eyes (Figure 4).

2. The host cornea was thinner than the graft at the grafthost junction. Graft step was detected in 15 eyes $(21.4 \%)$ and all of these eyes had keratoconus as a preoperative diagnosis (Figure 5).

3. The host cornea was thicker than the transplanted button. The host step was present in 14 (20\%) eyes, of which ten had PBK, two had traumatic scars, and two had Fuchs' dystrophy as the preoperative diagnosis (Figure 6).
A

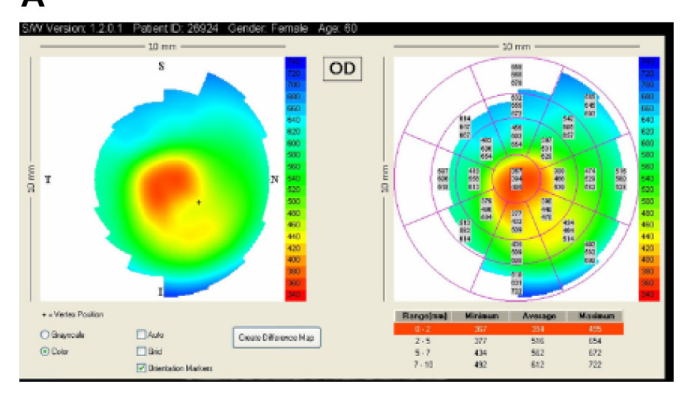

C

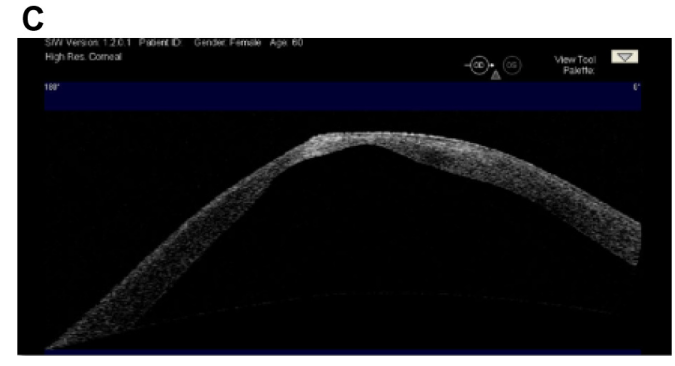

B

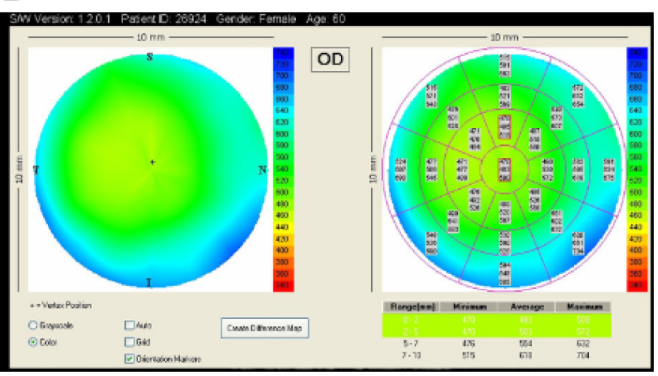

D

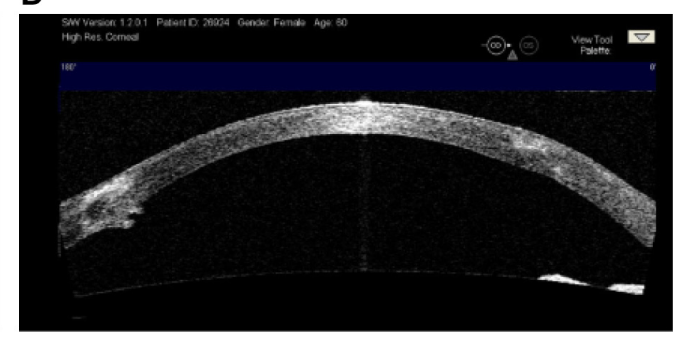

Figure I (A) The preoperative map of a 59-year old female patient with keratoconus in her right eye. The thinnest corneal pachymetry was measured as 367 microns. (B) Improved pachymetry map of the same eye one year after penetrating keratoplasty. (C) Preoperative AS-OCT demonstrating apical scar and significant posterior keratoconus. (D) Postoperative AS-OCT image of the cornea with well-apposed graft.

Abbreviations: AS-OCT, anterior segment optical coherence tomography; OD, oculus dexter. 


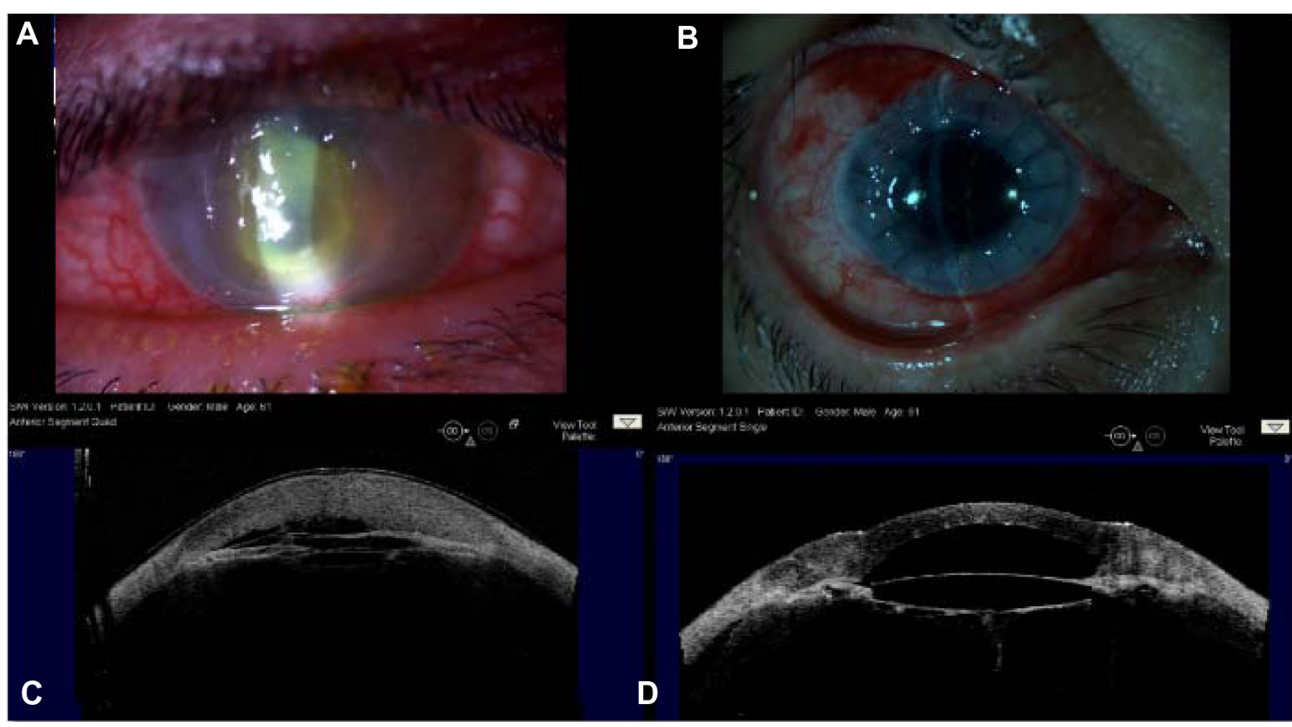

Figure 2 A 61 year-old male patient, who had a complicated cataract surgery elsewhere 3 months ago, presented with severe visual loss and pain; the visual acuity was 20/2000.

Notes: The slit-lamp examination showed a totally opaque cornea with a central corneal ulcer. (A) The details of the anterior chamber could not be visualized. (B) The upper right image is the slit-lamp photograph taken in the early postoperative period after PKP. (C) The AS-OCT image $\left(180^{\circ}-0^{\circ}\right)$ revealed stromal thickening, retrocorneal membrane, adhesions in the iridocorneal angle, and posterior chamber IOL. (D) The AS-OCT image demonstrates improved anterior segment relations. Note the protrusion of the wound site at the graft-host junction.

Abbreviations: AS-OCT, anterior segment optical coherence tomography; IOL, intraocular lens; PKP, penetrating keratoplasty.

4. Membrane-like remnants or tags arising from the host side of the wound edge were occasionally identified in the scans; they were not related to anterior synechia and/or retrocorneal membranes (Figure 7). Tags were present in one cross-section of four eyes (5.7\%), in two cross-sections of four eyes (5.7\%), in three cross-sections of three eyes (4.3\%), and not detectable in the rest of the eyes (84.3\%).

5. A gap formation at the graft-host junction was rare; it was observed in one cross-section of five eyes (7.2\%) (Figure 8).

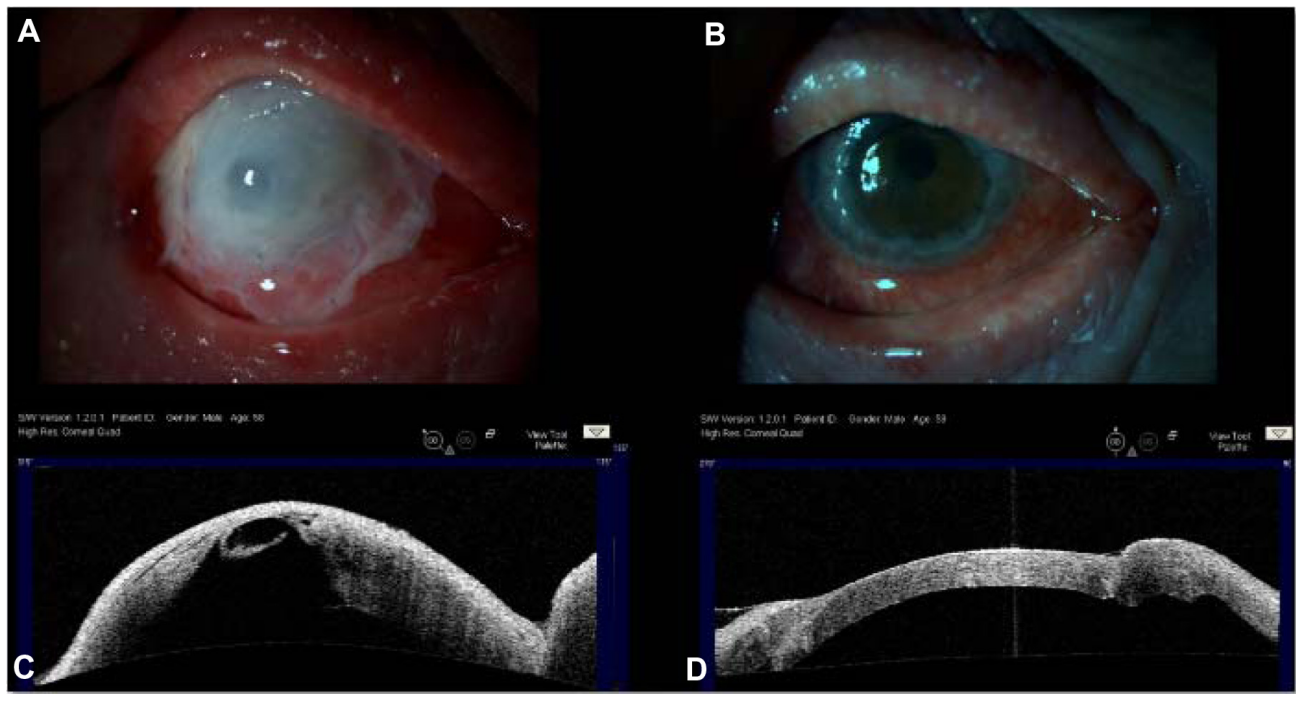

Figure 3 (A) Slit-lamp photograph of the right eye of a 57-year-old male patient with amniotic membrane transplantation due to graft abscess (PKP I5 years ago). (B) The visual acuity was light perception at the time of presentation. Slit-lamp photograph I month after regraft. The BSCVA was $20 / 200(-3.0-2.75 \times 40)$. (C) A high-resolution AS-OCT image reveals an extremely thinned central cornea with descemetocele and retrocorneal membrane. The amniotic membrane layers smoothly cover the central region of the cornea with imminent perforation. (D) High resolution AS-OCT image of the eye I month after PKP surgery.

Abbreviations: PKP, penetrating keratoplasty; AS-OCT, anterior segment optical coherence tomography; BSCVA, best spectacle-corrected visual acuity. 


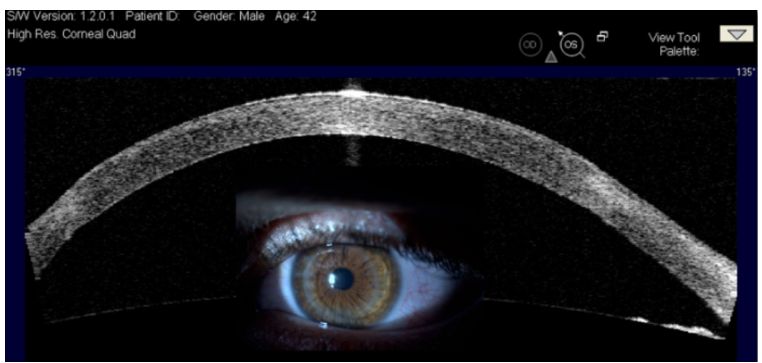

Figure 4 A 42-year-old male patient had penetrating keratoplasty in his left eye 9 years earlier due to advanced keratoconus.

Notes: BSCVA was 20/25 $(+2.0-3.0 \times 90)$ at his last visit. High-resolution AS-OCT (image from $315^{\circ}-135^{\circ}$ ) shows a well apposed graft-host relation.

Abbreviations: OD, oculus dexter; OS, oculus sinister; BSCVA, best spectaclecorrected visual acuity; AS-OCT, anterior segment optical coherence tomography.

6. A hill-like protrusion of the wound site from both outer and inner corneal surfaces was observed only in the early postoperative period (0-3 months). We had 17 patients with preoperative and early postoperative AS-OCT findings (Figure 9). In this subgroup, the finding was observed in ten eyes and became less significant over the course of time.

The graft-host junction variations described above are a classification concerning only the corneal wound site of all of the eyes. On the other hand, we have observed additional important AS-OCT findings in problematic cases such as eyes with anterior synechia, graft rejection, graft failure, and corneal ulcer.

\section{Postoperative complications Graft rejection}

In three eyes with acute graft rejection, high resolution AS-OCT showed stromal thickening and undulation of the posterior corneal surface. After treatment with systemic and

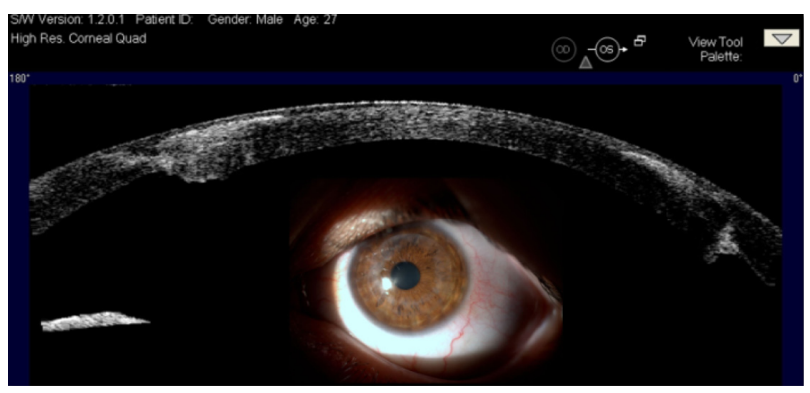

Figure 5 A 27-year-old male patient had penetrating keratoplasty in his left eye 2.5 years earlier; the preoperative diagnosis was also keratoconus.

Notes: BSCVA was 20/30 $(+4.0-2.50 \times 175)$ for this eye at his last visit. Highresolution AS-OCT (image from $180^{\circ}-0^{\circ}$ ) demonstrates that the anterior corneal surface is uniform. A significant graft step is seen at both graft-host junctions with highly reflective regions in the anterior stroma of the graft due to fibrosis.

Abbreviations: OD, oculus dexter; OS, oculus sinister; BSCVA, best spectaclecorrected visual acuity; AS-OCT, anterior segment optical coherence tomography.

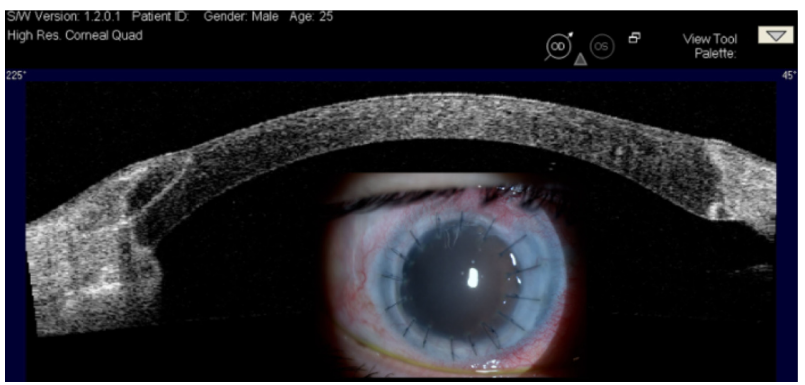

Figure 6 Left eye of a 25-year-old male patient I year after penetrating keratoplasty.

Notes: The preoperative diagnosis was bullous keratopathy after vitrectomy and silicone oil injection for traumatic retinal detachment. A high-resolution AS-OCT (image from $225^{\circ}-45^{\circ}$ ) image shows that the host cornea is thicker on one side $\left(225^{\circ}\right)$ where a suture trace is also visible. The graft-host junction at $45^{\circ}$ has better wound adaptation with a slight protrusion.

Abbreviations: OD, oculus dexter; OS, oculus sinister; AS-OCT, anterior segment optical coherence tomography.

topical steroids, the undulations diminished and the stroma became thinner (Figure 10). In two patients with chronic graft failure, stromal thickening of the cornea was observed, and the posterior corneal surface was smooth.

\section{Anterior synechia}

Peripheral anterior synechia (PAS) was observed pre- and intraoperatively in 14 eyes. Synechiolysis was performed as much as possible during each operation (Figure 6), but complete synechiolysis could not be achieved in four eyes due to the long duration of this pathological change. The first patient had PBK due to iris-fixated phakic IOL, the second patient had PBK due to anterior chamber IOL, the third patient had sclerocornea, and the last patient had a traumatic scar due to an explosion as the preoperative diagnosis. These patients were under topical antiglaucomatous treatment and had previously experienced rejection

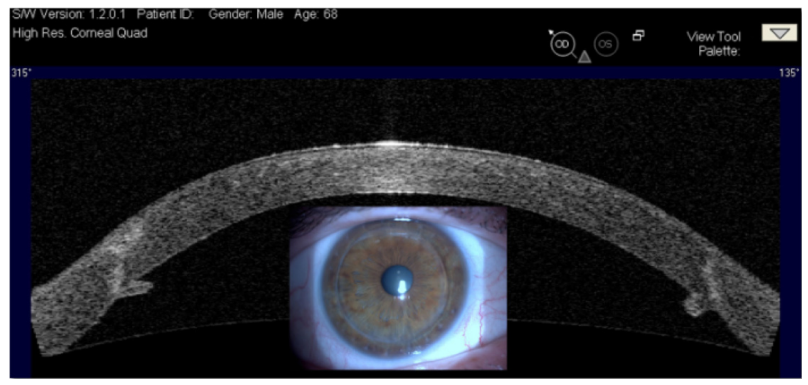

Figure 7 A 68-year-old male patient had penetrating keratoplasty in his right eye 8 years earlier.

Notes: The preoperative diagnosis was keratoconus. BSCVA was 20/25 $(+1.0-1.0$ $\times 125$ ) for this eye at the final visit. A high-resolution AS-OCT (image from $315^{\circ}-135^{\circ}$ ) image demonstrates tags that are present at the internal surface of the wound edge of both cross-sections.

Abbreviations: OD, oculus dexter; OS, oculus sinister; BSCVA, best spectaclecorrected visual acuity; AS-OCT, anterior segment optical coherence tomography. 


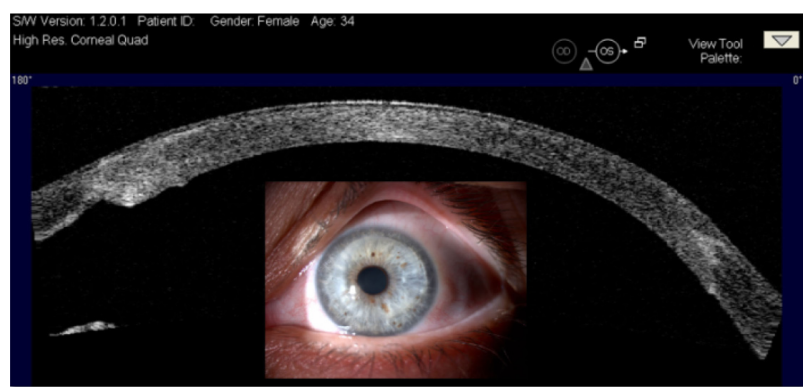

Figure 8 A 34-year-old female patient had penetrating keratoplasty in her left eye 5 years earlier due to advanced keratoconus.

Notes: The manifest refraction was $(-1.0-2.50 \times 20)$ and BSCVA was $20 / 30$ for this eye at her last visit. A gape of the posterior corneal surface at graft-host junction $\left(180^{\circ}\right)$ can be observed in the high-resolution AS-OCT image.

Abbreviations: OD, oculus dexter; OS, oculus sinister; BSCVA, best spectaclecorrected visual acuity; AS-OCT, anterior segment optical coherence tomography.

episodes in the past with loss of graft clarity to some extent, but none of them required a regraft.

\section{Corneal ulcer}

Three cases with severe keratitis were evaluated by AS-OCT. The first patient had a central corneal ulcer as a result of PBK and this patient was examined both preoperatively and after PKP during his follow-up. Figure 8 demonstrates both slit-lamp examination and AS-OCT findings of this patient.
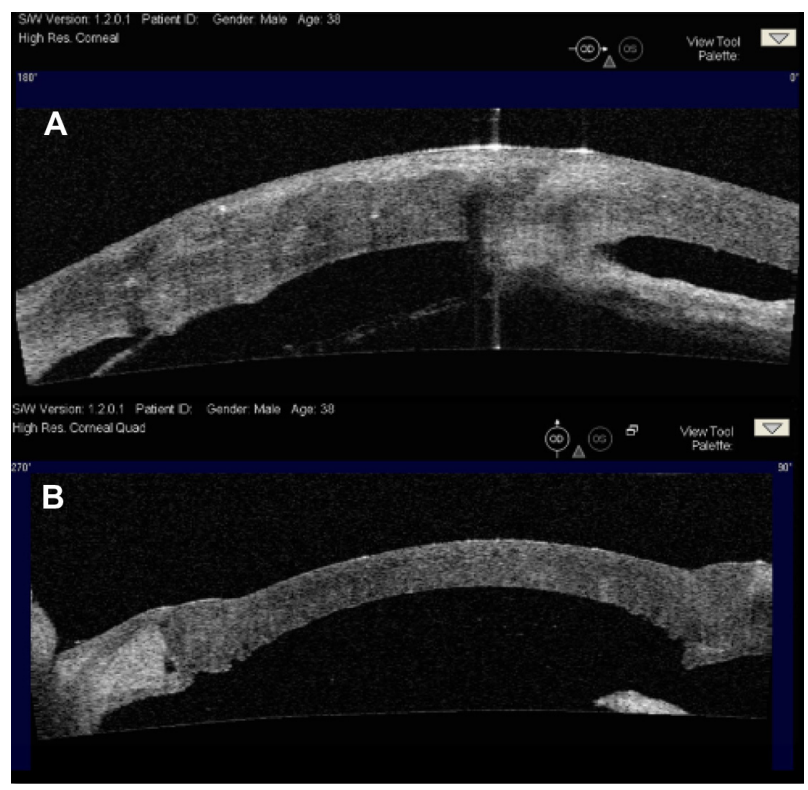

Figure 9 AS-OCT image of the right-eye of a 38-year-old male patient after penetrating trauma and postoperatively.

Notes: (A) High resolution AS-OCT image of the right eye of a 38-year-old male patient 2 months after penetrating trauma. (B) The $180^{\circ}$ image demonstrates a highly thickened cornea, anterior synechia to the corneal wound, and retrocorneal membrane. Early postoperative image of the same eye after PKP.

Abbreviations: OD, oculus dexter; OS, oculus sinister; AS-OCT, anterior segment optical coherence tomography; PKP, penetrating keratoplasty.
The second patient was an 81-year-old female who had PKP due to PBK and developed a peripheral corneal ulceration.

The third patient with atopic dermatitis who had bilateral PKP 15 years earlier followed by phacoemulsification and IOL implantation presented with a total corneal abscess of the right eye. It was impossible to evaluate the posterior cornea and anterior chamber by slit-lamp examination. The patient was monitored by AS-OCT as part of his daily follow-up during his challenging course of treatment. The infection reduced with intensive topical antimicrobial therapy, but both slit-lamp examination and the AS-OCT revealed imminent perforation. An urgent transplantation of the amniotic membrane was performed, and 10 days later he received a regraft (Figure 9).

\section{Discussion}

AS-OCT is a recently developed imaging technique customized for anterior segment evaluation. This novel device allows cross-sectional, clear visualization and accurate in-depth imaging, enabling micrometric measurements of the cornea and the anterior chamber. It is also capable of demonstrating the anterior segment structures that are otherwise not visible during slit-lamp examination due to opaque cornea. ${ }^{4,17}$

On the basis that AS-OCT might provide additional information regarding graft-host relations, we started using AS-OCT routinely in candidates for PKP, as well as in patients who had had this operation before this device was available for clinical use. In our study, we evaluated donorhost relations and complications after PKP by AS-OCT. Ideal graft-host relations of the internal wound site were observed in more than $50 \%$ of the eyes. We have seen that graft-host junction variabilities mostly depended on the preoperative pathological characteristics of the host cornea and anterior chamber structures. For example, cases with thinner host cornea at the graft-host junction were all keratoconic eyes. Thicker host cornea, however, was almost always related to preoperative corneal pathologies, which had caused corneal thickening such as PBK.

The tags arising from the host side of the wound junction were considered to belong to the posterior stroma and Descemet's membrane. In PKP, during the trephination of the host cornea, a full thickness 360-degree trephination along the circumference does not always occur, and the cutting process is completed with the aid of corneal scissors. We are of the opinion that the Descemet's membrane rests are the result of incomplete trephination and manual completion by scissors. Tags that were seen in our scans were rather small 


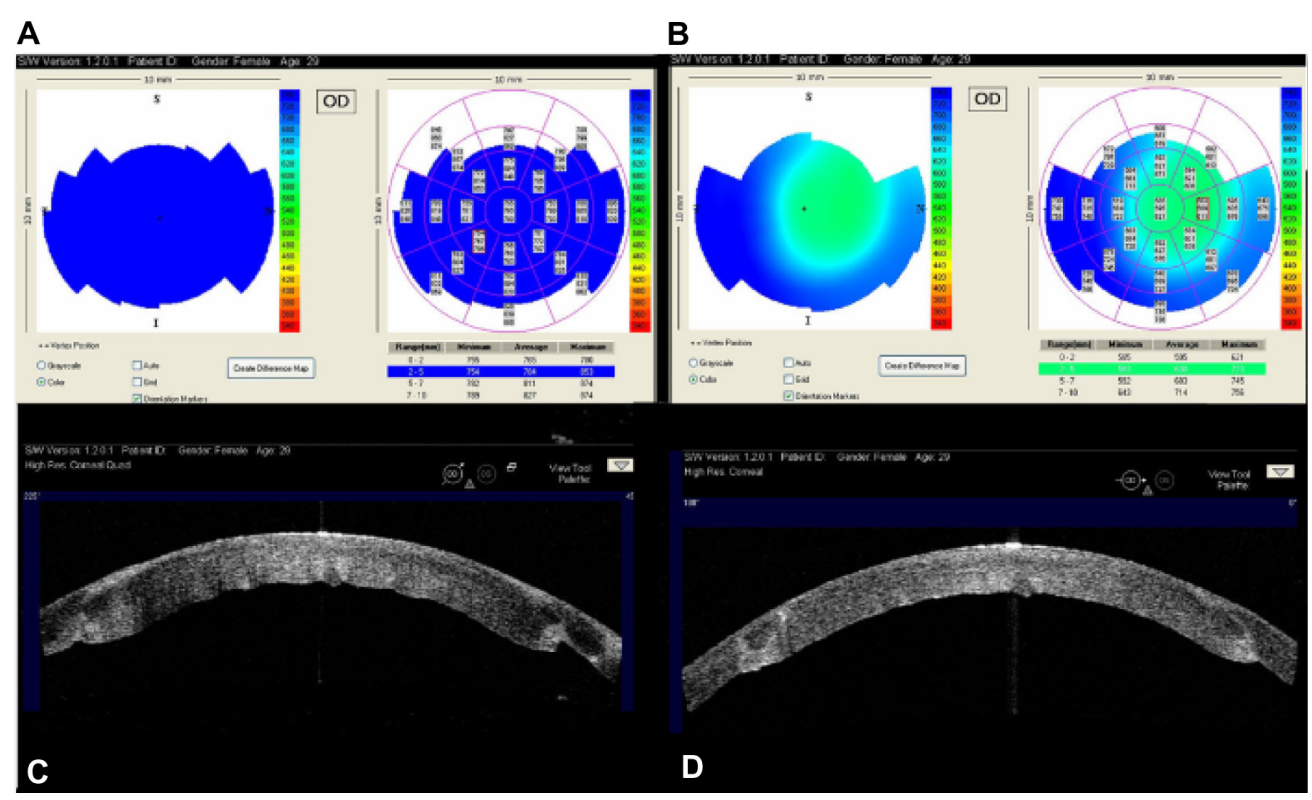

Figure 10. High resolution AS-OCT images and pachymetry maps of the right eye of a 28 -year-old female patient 3 years after penetrating keratoplasty. The condition was prediagnosed as acute graft rejection.

Notes: (A) The pachymetry map demonstrates corneal thickening due to edema. (B) The pachymetry map after treatment. (C) The corneal stroma is thickened and the posterior corneal surface shows undulation before treatment. (D) After treatment, the posterior corneal surface is more uniform and the stromal thickness has reduced. Abbreviations: AS-OCT, anterior segment optical coherence tomography; OD, oculus dexter; OS, oculus sinister.

and did not cause anterior synechia. They were also not related to retrocorneal membranes.

Kaiserman et $\mathrm{al}^{18}$ retrospectively evaluated graft-host sections in 27 eyes with full-thickness grafts by AS-OCT. They showed that malapposition of the internal graft-host junction after PKP is common and present in more than half $(60.8 \%)$ of the sections examined, which is similar to our findings.

Jhanji et a ${ }^{19}$ studied the wound configuration after PKP by AS-OCT and observed the smooth anterior graft-host junction in all cases. However, the authors reported common posterior graft-host malapposition in the form of host "ledges" in keratoconic eyes and graft "steps" in eyes with preoperative endothelial dysfunction.

Graft rejection is a serious postoperative complication of PKP. Early treatment of this condition is critical to prevent irreversible graft failure. Therefore, prompt recognition is important for the survival of an affected corneal graft. When we are presented with distinct corneal edema after PKP, AS-OCT may help us in the differential diagnosis. We have observed undulations of the posterior corneal surface in high resolution AS-OCT scans of patients with acute graft rejection. On the other hand, AS-OCT scans of patients with graft failure demonstrated stromal thickening though the posterior corneal surface was smooth and parallel to the anterior corneal surface. Our view is that undulations of the posterior corneal surface seen in high resolution AS-OCT images may be a specific feature of early graft rejection.

Severe keratitis and corneal ulceration are other important issues where AS-OCT may be complementary in the treatment regime and follow-up. In eyes with severe corneal ulcer where urgent PKP may be necessary, AS-OCT evaluation during the treatment course and waiting period for donor cornea can be extremely helpful. The thickness of the cornea at the ulcer base could be estimated, and the perforation risk could be assessed quickly and accurately. In our case, following amniotic membrane transplantation (Figure 9), we were able to see that the infection did not spread in the anterior chamber. Similarly, we monitored the patient after regraft in order to be able to diagnose the recurrence of the infection and/or other related complications.

Iridocorneal synechia are changes that need attention in keratoplasty patients. Peripheral anterior synechia is important because it may cause secondary-angle closure glaucoma and lead to graft failure. Iridocorneal adhesions closer to the wound site may also enhance the occurrence of graft rejection. ${ }^{20}$ Of our four patients with anterior synechia in the postoperative period, two of them had optic atrophy due to secondary glaucoma. The existence of postoperative synechia may not always be visible by slit-lamp; in this case, AS-OCT may be helpful in recognizing the lesions. Chua et $\mathrm{al}^{5}$ reported on the use of AS-OCT in assessing secondary glaucoma in a case after PKP, and concluded that AS-OCT 
was a useful investigating tool that enabled better evaluation of the structures within the anterior segment.

In our smaller subgroup of eyes with preoperative evaluation, we have seen that AS-OCT assessment was important in planning the surgical technique. In eyes with totally opaque corneas, AS-OCT was complementary in evaluating the posterior cornea, anterior chamber configuration, and structures. In eyes with corneal opacities and semiopaque corneas, the depth of the pathology could be identified by highresolution AS-OCT for the final indication of keratoplasty: lamellar or penetrating. The presence of cataracts and/or IOL, as well as abnormalities of anterior segment elements could also be evaluated. Therefore, it may be concluded that ASOCT assessment was helpful presurgically in determining the strategy and indication of lens-cataract-IOL surgery where combined procedures with PKP were required.

Lim et $\mathrm{al}^{9}$ published a report on the usefulness of AS-OCT in imaging the layers of the cornea after anterior and posterior lamellar keratoplasty procedures (seven eyes) and in the management of surgical complications after such procedures. They found that AS-OCT images were able to provide valuable information on donor apposition, Descemet's membrane detachment after deep anterior lamellar keratoplasty, posterior lamellar dislocation, primary graft failure, and pupillary block after Descemet's stripping automated endothelial keratoplasty. Our study followed a similar concept, but we investigated full thickness grafts. ${ }^{9}$

Not only high-resolution but standard anterior segment scans are also noteworthy in examining eyes with PKP. Anterior segment quad images provide an overall view of the anterior segment and show the relation of the transplanted cornea to the anterior segment structures (ie, iridocorneal angle, lens, or IOL). The overall configuration of the anterior chamber can be better visualized by this scan modality. It is also possible to change the scan direction in any meridian providing a complete, dynamic, 360-degree evaluation. This issue is especially important in the preoperative examination of eyes with opaque corneas.

In spite of these advantages, there is a disadvantage of AS-OCT that needs to be mentioned. Due to the backscattering effect of the posterior iris pigment epithelium, imaging of structures in the posterior chamber is poor. ${ }^{21}$ Ultrasound biomicroscopy has a better imaging quality posterior to the iris pigment epithelium, but it requires contact with the ocular surface.

In conclusion, AS-OCT can be used for diagnostic purposes and for monitoring treatment outcomes of patients with PKP, and it can also be used for keratoplasty candidates. In eyes with a complicated pre- and postoperative course, ASOCT may reveal additional important findings on the information gathered by slit-lamp examination. A high-resolution cornea modality can be used to visualize the posterior cornea and iridocorneal angle beyond a nontransparent cornea. In the diagnosis and management of graft complications, AS-OCT may provide valuable information and safer follow-up. It is also useful and complementary in the diagnosis and management of other postoperative complications following PKP.

\section{Disclosure}

This study was presented as a poster at the 15th European Society of Cataract and Refractive Surgeons Winter Meeting, February 18-20, 2011, Istanbul, Turkey. None of the authors has a financial or proprietary interest in any material or method mentioned. The authors report no other conflicts of interest in this work.

\section{References}

1. Chen WL, Hu FR, Wang IJ. Changing indications for penetrating keratoplasty in Taiwan from 1987 to 1999. Cornea. 2001;20(2): 141-144.

2. Frost NA, Wu J, Lai TF, Coster DJ. A review of randomized controlled trials of penetrating keratoplasty techniques. Ophthalmology. 2006;113(6):942-949.

3. Huang D, Li Y, Radhakrishnan S, Chalita MR. Corneal and anterior segment optical coherence tomography. In: Schuman JS, Puliafito CA, Fujimoto JG, editors. Optical Coherence Tomography of Ocular Diseases. 2nd ed. Thorofare, NJ: SLACK Incorporated; 2004:663-673.

4. Ramos JL, LiY, Huang D. Clinical and research applications of anterior segment optical coherence tomography - a review. Clin Experiment Ophthalmol. 2009;37(1):81-89.

5. Chua J, Mehta JS, Tan DT. Use of anterior segment optical coherence tomography to assess secondary glaucoma after penetrating keratoplasty. Cornea. 2009;28(2):243-245.

6. See JL. Imaging of the anterior segment in glaucoma. Clin Experiment Ophthalmol. 2009;37(5):506-513.

7. Kucumen RB, Yenerel NM, Gorgun E, Kulacoglu DN, Dinc UA, Alimgil ML. Anterior segment optical coherence tomography measurement of anterior chamber depth and angle changes after phacoemulsification and intraocular lens implantation. J Cataract Refract Surg. 2008;34(10):1694-1698.

8. Fine IH, Hoffman RS, Packer M. Profile of clear corneal cataract incisions demonstrated by ocular coherence tomography. $J$ Cataract Refract Surg. 2007;33(1):94-97.

9. Lim LS, Aung HT, Aung T, Tan DT. Corneal imaging with anterior segment optical coherence tomography for lamellar keratoplasty procedures. Am J Ophthalmol. 2008;145(1):81-90.

10. Kucumen RB, Dinc UA, Yenerel NM, Gorgun E, Alimgil ML. Immediate evaluation of the flaps created by femtosecond laser using anterior segment optical coherence tomography. Ophthalmic Surg Lasers Imaging. 2009;40(3):251-254.

11. Kucumen RB, Yenerel NM, Gorgun E, Dinc UA. Anterior segment optical coherence tomography findings of acute hydrops in a patient with keratoconus. Ophthalmic Surg Lasers Imaging. 2010;9: $1-3$.

12. Tarnawska D, Wylegala E. Monitoring cornea and graft morphometric dynamics after descemet stripping and endothelial keratoplasty with anterior segment optical coherence tomography. Cornea. 2010;29(3): 272-277. 
13. Wylegala E, Dobrowolski D, Nowińska A, Tarnawska D. Anterior segment optical coherence tomography in eye injuries. Graefes Arch Clin Exp Ophthalmol. 2009;247(4):451-455.

14. Nieuwendaal CP, van Velthoven ME, Biallosterski C, et al. Thickness measurements of donor posterior disks after descemet stripping endothelial keratoplasty with anterior segment optical coherence tomography. Cornea. 2009;28(3):298-303.

15. Yoo SH, Kymionis GD, Deobhakta AA, et al. One-year results and anterior segment optical coherence tomography findings of descemet stripping automated endothelial keratoplasty combined with phacoemulsification. Arch Ophthalmol. 2008;126(8):1052-1055.

16. Di Pascuale MA, Prasher P, Schlecte C, et al. Corneal deturgescence after Descemet stripping automated endothelial keratoplasty evaluated by Visante anterior segment optical coherence tomography. Am J Ophthalmol. 2009;148(1):32-37. e1.
17. Baikoff G, Lutun E, Ferraz C, Wei J. Static and dynamic analysis of the anterior segment with optical coherence tomography. J Cataract Refract Surg. 2004;30(9):1843-1850.

18. Kaiserman I, Bahar I, Rootman DS. Corneal wound malapposition after penetrating keratoplasty: an optical coherence tomography study. Br J Ophthalmol. 2008;92(8):1103-1107.

19. Jhanji V, Constantinou M, Beltz J, Vajpayee RB. Evaluation of posterior wound profile after penetrating keratoplasty using anterior segment optical coherence tomography. Cornea. 2011;30(3):277-280.

20. Tragakis MP, Brown SI. The significance of anterior synechiae after corneal transplantation. Am J Ophthalmol. 1972;74(3):532-533.

21. Radhakrishnan S, Rollins AM, Roth JE, et al. Real-time optical coherence tomography of the anterior segment at $1310 \mathrm{~nm}$. Arch Ophthalmol. 2001;119(8):1179-1185.
Clinical Ophthalmology

\section{Publish your work in this journal}

Clinical Ophthalmology is an international, peer-reviewed journal covering all subspecialties within ophthalmology. Key topics include: Optometry; Visual science; Pharmacology and drug therapy in eye diseases; Basic Sciences; Primary and Secondary eye care; Patien Safety and Quality of Care Improvements. This journal is indexed on

Submit your manuscript here: http://www.dovepress.com/clinical-ophthalmology-journal

\section{Dovepress}

PubMed Central and CAS, and is the official journal of The Society of Clinical Ophthalmology (SCO). The manuscript management system is completely online and includes a very quick and fair peer-review system, which is all easy to use. Visit http://www.dovepress.com/ testimonials.php to read real quotes from published authors. 